\title{
Replacing vascular corrosion casting by in vivo micro-CT imaging for building 3D cardiovascular models in mice
}

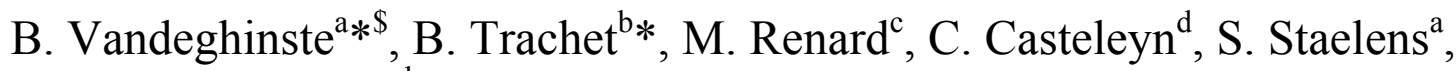 \\ B. Loeys ${ }^{c}$, P. Segers ${ }^{b}$ and S. Vandenberghe ${ }^{a}$ \\ ${ }^{a}$ IBITECH-MEDISIP, Ghent University - IBBT, Ghent, Belgium; ${ }^{b}$ IBITECH-bioMMeda, \\ Ghent University, Ghent, Belgium; ' Centre for Medical Genetics, Ghent University, \\ Ghent, Belgium; ' Department of Morphology, Ghent University, Merelbeke, Belgium \\ *These authors contributed equally to this work. \\ ${ }^{\$}$ Corresponding author. \\ Email: Bert.Vandeghinste@UGent.be \\ Telephone: +3293324322 \\ Fax: +32 93324159
}

Purpose: The purpose of this study was to investigate if in vivo micro-CT is a reliable alternative to micro-CT scanning of a vascular corrosion cast. This would allow to study the early development of cardiovascular diseases.

Procedures: Datasets using both modalities were acquired, segmented and used to generate a 3D geometrical model from 9 mice. As blood-pool contrast agent Fenestra VC-131 was used. Batson's No. 17 was used as casting agent. CFD simulations were performed on both datasets to quantify the difference in wall shear stress (WSS).

Results: Aortic arch diameters show 30 to $40 \%$ difference between the Fenestra VC-131 and the casted dataset. The aortic arch bifurcation angles show less than $20 \%$ difference between both datasets. Numerically computed WSS showed a $28 \%$ difference between both datasets.

Conclusions: Our results indicate that in vivo micro-CT imaging can provide an excellent alternative for vascular corrosion casting. This enables follow-up studies.

Keywords: Fenestra; in vivo; micro-CT; mice; vascular corrosion casting; 3D model; wall shear stress 


\section{Introduction}

Mouse models are often used for medical research in the cardiovascular field, since they can provide valuable information on the development and progression of cardiovascular pathologies within a reasonable timeframe. Genetically modified mice have been used to study e.g. abdominal aortic aneurysm formation [1] or atherosclerosis [2, 3], but also to study more specific genetic disorders such as the Marfan and Loeys-Dietz syndrome [4, $5,6]$.

Hemodynamic factors and blood flow patterns have been linked to the genesis and development of atherosclerotic cardiovascular disease [7, 8] and to the growth and progression of aneurysms [9]. Computational Fluid Dynamics (CFD) are often used to simulate the flow patterns inside the aorta, both in animal and in human models, in an attempt to elucidate the role of these biomechanical actors/stimuli in the early disease stages. An important parameter in this respect is the tangential shear force exerted by moving blood along the axis of flow (wall shear stress or WSS). It would be very useful to be able to perform realistic CFD simulations on (genetically modified) animal models.

In order to perform such CFD simulations, reliable 3D computer models of the arterial system are needed. Most studies that performed CFD simulations in mouse models in the past created a geometrical model by in vitro micro-CT scanning of a plastic replica of the arterial system obtained by vascular corrosion casting [10, 11, 12]. However, this technique requires sacrificing the animals and thus excludes follow-up studies. If one wants to study the influence of local hemodynamics on disease development, it is mandatory to perform follow-up studies in which the hemodynamic situation pre and post disease development can be compared. To perform such studies, 
alternative in vivo imaging techniques permitting scans of the same animal at multiple time points are indispensable. In vivo imaging with micro-CT could be such an alternative, but traditional iodine-based contrast agents used in human medicine (needed to differentiate the aorta from surrounding tissues) cannot be used for mice since they are cleared through the murine renal system within one minute, whereas micro-CT scanners typically have a total acquisition time of at least 5 to 10 minutes for an acceptable level of image quality. Fenestra VC-131 ${ }^{\circledR}$ (Advanced Research Technologies Inc., Saint Laurent, Canada) is a new contrast agent developed for cardiovascular imaging in mice that has a slow uptake in the liver, enabling a sufficient time period of contrast-enhanced imaging.

The goal of our study was to investigate whether reliable and accurate 3D geometrical models of the murine aortic arch could be reconstructed using in vivo microCT with this contrast agent, Fenestra VC- $131^{\circledR}$. We compared geometrical models obtained using a contrast injection followed by an in vivo micro-CT scan with models obtained afterwards from the same animals using vascular corrosion casting followed by an in vitro micro-CT scan. 


\section{Material and methods}

A total of 9 wild-type mice were used in the experiments, with body weights ranging from 14 to 35 gram (tables 1 and 2). The animals were anesthetized and scanned in vivo after administration of the contrast agent. They were subsequently casted via the abdominal aorta with $2 \mathrm{ml}$ of Batson's No. 17 casting solution. The resulting plastic replicas of the arterial system were scanned in vitro using micro-CT. Both data sets were segmented and compared. The Ghent University ethical committee approved all animal experiments (ECD 07/20).

\section{In vivo : Fenestra $V C-131^{\circledR}$}

Fenestra VC- $131^{\circledR}$ binds iodine particles $(50 \mathrm{mg} / \mathrm{ml})$ on lipids, which ensures clearance in the liver, leading to 4 hours of contrast enhanced visualisation time. Previous research by Mukundan et al. [13] has shown that Fenestra VC-131 ${ }^{\circledR}$ induces no significant risk on renal toxicity. Nine 5 to 27 week-old animals were put on a diet of cooked yam 24 hours before the experiment and received an intraperitoneal injection of $100 \mu \mathrm{L}$ physiological fluid one hour before the start of the experiment. Anecdotal evidence gathered from small experiments (not depicted here) has shown that these measures help to prevent dehydration, which can be caused by the use of Fenestra VC- $131^{\circledR}$. Fifteen minutes after the administration of $0.015 \mathrm{ml} /$ gram Fenestra VC- $131^{\circledR}$ through the tail vein with a bolus injection, the animals were anesthetized with an $8 \mu \mathrm{L} /$ gram IP injection of a mixture of $1.05 \mathrm{ml}$ ketamine (Ketamine 1000, CEVA Santé Animale, Brussel, Belgium), $0.3 \mathrm{ml}$ xylazine (Xyl-M $2 \%$, VMD, Arendonk, Belgium) and $3.4 \mathrm{ml}$ physiological fluid. The animals were scanned after 10 minutes of anaesthesia, as optimal contrast is achieved 25 
minutes after administering Fenestra ${ }^{\circledR}$ [14]. The 25 minute total waiting time also reduced the chances on spasms and other irregularities affecting imaging quality. The mice were then placed on a heated pad $\left(30^{\circ} \mathrm{C}\right)$.

\section{micro-CT}

The animals were scanned in a GE FLEX Triumph CT scanner (Gamma Medica-Ideas, Northridge, USA) with the following acquisition parameters: $50 \mu \mathrm{m}$ focal spot, $2 \times 2$ detector binning, 2048 projections over 360 degrees, 3.5 times magnification and $70 \mathrm{kVp}$ tube voltage. Using a blank air scan, the ideal tube current was determined by increasing the current until the detector response saturated. This ideal tube current was determined at $180 \mu \mathrm{A}$ for a $70 \mathrm{kVp}$ tube voltage. The gantry rotated continuously, providing faster acquisition compared to step-and-shoot mode. This results in a $33.81 \mathrm{~mm}$ transverse field of view, a theoretical spatial resolution of $46 \mu \mathrm{m}$ and a scanning time of 8.53 minutes. The projections were reconstructed with proprietary software (Cobra EXXIM, EXXIM Computing corp., Livermore, USA) using a Feldkamp-type algorithm with Parker's weighting function [15] in a $512 \times 512 \times 512$ matrix with a $50 \mu \mathrm{m}$ voxel size.

\section{Gating}

In addition to standard reconstruction, we also employed a retrospective respiratory gating method. The idea was first proposed by Ford et al. [16, 17]. A region of interest (ROI) was selected on the projection images containing both the diaphragm and the lungs of the animal. This allows us to compute the centre of mass $(\mathrm{CM})$ of the ROI in one projection. Now the projection can be classified as in peak expiration or peak inspiration phase according to the location of the CM. After classification of all projections, 820 
minimal lung volume projections $(70 \mathrm{kVp}, 180 \mu \mathrm{A})$ were selected and reconstructed while the ungated images were reconstructed from a 2048 projections dataset.

Reconstruction of the reduced dataset requires modification of the Feldkamp reconstruction algorithm by incorporating Parker's weighting function, to account for the non-uniform angles of the projections.

\section{In vitro: Vascular corrosion casting}

For each of the 9 animals scanned in vivo, a corrosion cast was created 1 week after acquiring the micro-CT images. When the health of the animal did not permit waiting one week, the animal was casted as soon as possible. Batson's No. 17 (Polysciences, Inc., Warrington, USA) was used as casting agent. This agent causes little shrinkage, can still fill the smallest vessels and delivers mechanically stable casts.

First, the mice were euthanized by an inhaled $\mathrm{CO}_{2}$ overdose. After making an abdominal incision, the abdominal aorta was located. Special care was taken to prevent rupture to vessels or organs, as this would greatly reduce the quality of the cast. A catheter was then placed in the abdominal aorta in retrograde direction and the arterial system was perfused with a ready-made mixture of Batson's No. 17. Complete filling of the vascular system was indicated by the appearance of red intravascular polymer shining through the skin of the toes and nose [18]. After this perfusion the animal was placed in a cold water bath to avoid tissue damage that might occur during the exothermic curing process.

After 30 minutes, the preparation was placed for 3 days in a bath containing a macerating solution of $20 \% \mathrm{KOH}$ at room temperature. Subsequently, the cast was cleaned in distilled water and dried. 
Scans were generated from these casts by the same micro-CT scanner as used in the in vivo experiments, with parameters: $2 \times 2$ binning, 2048 projections, $70 \mathrm{kVp}(180$ $\mu \mathrm{A})$ and magnification $3 \mathrm{x}$. The casts were placed in an acrylic tube to prevent the cast from motion due to airflow.

\section{Segmentation}

Each dataset (one from in vivo experiments, one from casting the same animal) was segmented manually in Mimics software (Materialise, Leuven, Belgium). Each part of the aortic arch (ascending aorta, aortic arch with its branches and the descending aorta) was manually thresholded in a first segmentation step, using different threshold levels for each part. This allows a more accurate segmentation, as every part has a distinct grey value on the images.

When the resulting segmentation was judged sufficiently accurate, a 3D geometric model was built according to this segmentation mask. This 3D model was smoothed in Mimics Remesher (Materialise, Leuven, Belgium), to remove unphysiological bulges and dents while care was being taken that no artificial shrinking of the model occurred. This resulted in a sufficiently smooth and simple 3D model usable for CFD simulations.

\section{Comparison}

The actual comparison between both modalities was computed in 4 different ways:

1) We determined the diameter of the 3D models on 6 different locations: ascending aorta, descending aorta, left subclavian artery, left common carotid artery, brachiocephalic trunk and the middle of the aortic arch between the left common 
carotid artery and the brachiocephalic trunk. Each diameter was then calculated as the mean over a sufficiently large area.

2) As second metric we considered the bifurcation angles. These were measured in the plane of the bifurcation as the angle between the centerline of the side branch and the centerline of the aortic arch. This provides insight in the angle and resistance encountered by the blood flow in moving from the aortic arch to a side branch.

3) As a third metric we considered a general distance metric between co-registered voxels on the models. First we co-registered every casting model with the in vivo data from the same animal, and then we used a colour code dependent on the distance between both 3D models.

4) Finally we performed CFD simulations on all 3D models to compare the influence of the imaging techniques on the resulting computed wall shear stresses (WSS) levels. All CFD simulations were performed as described in our earlier work [10]. In order to quantify the difference in WSS between the models, spatially averaged WSS (WSS $\mathrm{Wv}_{\mathrm{av}}$ ) was computed for each time-averaged model as well as the $95 \%$ WSS value $\left(\mathrm{WSS}_{95 \%}\right.$ ) as a marker of highest WSS values. 


\section{Results}

High-quality casts were obtained for 4 animals, which were scanned using 0.015 $\mathrm{ml} /$ gram Fenestra (mice 5, 6, 7 and 8 in table 1). A small proof of concept study has shown that the contrast increase by administering $0.015 \mathrm{ml} / \mathrm{gram}$ Fenestra VC- $131^{\circledR}$ (figures 1c and 1d) leads to an increase of $118 \pm 2$ gray values in the aortic arch, compared to a baseline image (figures 1a and 1b). The mean image noise in the baseline image was determined at 24.7 gray values. Casts from mice 1, 2, 3, 4 and 9 were not filled completely and contained air bubbles; these were thus not further used in the measurements. The time between casting and acquisition differed greatly between mice 7 and 8 and the other mice. Five out of 9 animals were sacrificed prematurely due to declining health of the animals and to limit animal suffering.

While casting the animals, some small effects were noticed: the liver was coloured more white than usual, some lymph nodes were opaque white and we noticed some mice had cornea oedema.

Measurements obtained from the 3D models generated from the scanned casts and in vivo images are shown in tables 2 and 3, showing aortic arch diameters and bifurcation angles. The diameters show a significant difference between in vivo and cast models. Using Fenestra VC- $131^{\circledR}$ this difference amounts from $30 \%$ up to $40 \%$ increase in diameter. The aortic arch diameters show an overall $31 \%$ difference $(31.9 \pm 1.8 \%)$, while the three bifurcation diameters show a larger difference variation $(35.7 \pm 7.4 \%)$. The bifurcation angles show a relatively small difference (less than 20\%) between the casts and the in vivo models.

Figure 2 shows a comparison between ungated slices (figures $2 \mathrm{a}$ and $2 \mathrm{c}$ ) and retrospective respiratory-gated slices (figures $2 \mathrm{~b}$ and $2 \mathrm{~d}$ ) for mouse 5 (table 1). All 4 
slices are shown in the same window level and size. The gated images show better delineation of the diaphragm and the ribs. The cardiovascular system was visualised by $0.015 \mathrm{ml} /$ gram Fenestra VC- $131^{\circledR}$ and showed no significant image quality increase due to respiratory gating. The increase in image noise due to the low angular sampling decreased the segmentation accuracy.

Figures 3 and 4 show the regression and Bland-Altman plots of the (pooled) aortic diameters and the aortic angles. The Pearson correlation coefficient equals 0.91 for the diameters (Standard Error of the Estimate $0.12 \mathrm{~mm}$ ) and 0.97 for the angles (SEE 11.13 degrees).

Figure 5 compares the general geometry in 3 different view angles of the $3 \mathrm{D}$ model built from mouse 6 using both modalities. Figure $\mathbf{6}$ shows the co-registered models from Fenestra ${ }^{\circledR}$ and the casts coloured with a green-red colouring scheme. Green was used to mark voxels with little distance; red was used when a large distance was measured. These colours were normalised and the different models can thus be compared. The measurements were obtained using a voxel to voxel comparison. These results show that the general geometry (aortic arch) of both the 3D cast and the 3D in vivo model are highly comparable. The descending aorta region shows the highest difference.

Figure 7 shows a comparison of the time-averaged WSS distribution for mouse 6 for both a casting and an in vivo model. The distribution of time-averaged WSS over the model surface is very similar: zones with high or low absolute values occur in the same region for both models. However, the in vivo models on average show lower absolute WSS values: the spatially- and time-averaged $\mathrm{WSS}_{\mathrm{av}}$ is $8.20 \pm 0.79 \mathrm{~Pa}$ for in vivo models and $10.52 \pm 2.52 \mathrm{~Pa}$ for the cast models. The time-averaged $95 \%$ percentile $\mathrm{WSS}_{95 \%}$ was 
$16.55 \pm 1.60 \mathrm{~Pa}$ for the in vivo models and $19.35 \pm 3.72 \mathrm{~Pa}$ for the cast models. 


\section{Discussion}

In this work, we proposed a method to visualise the aortic arch and the aortic arch bifurcations using micro-CT, providing an alternative for vascular corrosion casting. The resulting $3 \mathrm{D}$ geometry models from in vivo scans were compared to models of vascular corrosion casts and were found to be an accurate representation, although the diameters showed a significant difference.

Schambach et al. [19] have already used both a blood pool contrast agent $\left(\right.$ Fenestra $\left.^{\circledR}\right)$ and a bolus technique with a conventional contrast agent (Imeron ${ }^{\circledR}$ ). Their setup was different from ours, with the probe (and the animal) rotating horizontally in the course of the beam. This results in a shorter acquisition time, but also introduces movement artefacts and higher radiation doses (due to the larger magnification). This study clearly shows the need to address technical challenges still involved with in vivo micro-CT imaging of the cardiovascular system in mice. Willekens et al. [14] used Fenestra ${ }^{\circledR}$ and eXIA $160^{\circledR}$ to enhance liver and spleen contrast in mice, but did not study the effects on the arterial system or the difference between in vivo and ex vivo models.

The difference between in vivo and in vitro methods to obtain $3 \mathrm{D}$ models of murine vasculature has not been studied extensively in literature. Kratky et al. [20] have demonstrated Batson's No. 17 to induce a shrinking factor of 16-20\%, but did not compare in vitro with in vivo models. Casteleyn et al. [21] showed that the morphology of 3D models of the murine aortic arch (obtained using vascular corrosion casting) is very similar to the one of the human aortic arch (obtained in vivo). An alternative method to obtain 3D models of the murine arterial geometry in vivo is MRI imaging. Moore et al. [22] compared this technique to vascular corrosion casting and also found casts to have 
smaller diameters and bifurcation angles. However, small animal MRI is an expensive technique that is not widely available yet.

We found that retrospective respiratory gating is not necessary to get a high $3 \mathrm{D}$ model quality. The ribs and diaphragm are more clearly delineated, but the difference is not noticeable in the aorta or the aortic arch. Cardiac gating can be done prospectively, where the ECG signal is used to determine if a projection should be acquired at that specific moment. This requires a very short integration time on the detector, and an X-ray tube which can be switched on and off in the order of milliseconds. Leaving the X-ray tube on for the duration of the whole gated scan would deliver a radiation overdose to the small animal. This hardware was unavailable to us. The influence of cardiac gating was thus not studied in this work, but may be presented in future work.

The radiation dose for a one-minute fly-scan at $2 \mathrm{x} 2$ binning, $80 \mathrm{kVp}$ and $1.3 \mathrm{x}$ magnification was reported at 20-25 mGy. As the dose is proportional to the effective tube current-time product, we can suspect the dose for our protocol to be in the order of 100-150 mGy, low enough to allow for follow-up studies.

For Fenestra VC- $131^{\circledR}$ the minimal contrast dose needed to obtain sufficient contrast was determined beforehand in a small proof of concept study. A $0.015 \mathrm{ml} / \mathrm{gram}$ contrast dose induces a relatively high strain on the animals, as this accounts for $25 \%$ extra blood volume. The small health effects noticed in the liver and lymph nodes can with high probability be amounted to the use of Fenestra ${ }^{\circledR}$ and this high contrast volume, causing the high mortality rate.

When comparing the diameters (table 2), a general difference of 30 to $40 \%$ was measured. However, the correlation plot in figure 3 (right panel) and the Bland-Altman 
plot in figure 4 (right panel) show that the diameters of both modalities are well correlated $(\mathrm{r}=0.91)$ and there is no bias for low or high absolute values of the diameter. In literature vascular corrosion casts have been reported to shrink 16-20\% [20]. One might hypothesize that our casts have shrunk more, since the Batson's solution was injected by free-hand without manometric control of the injection pressure. However, according to Hodde [23] this technique gives consistently better results than injection with an injection apparatus. We therefore hypothesize that the extra 10 to $20 \%$ difference can be attributed to the increase in arterial pressure due to overfilling the animal during the in vivo scans, given the high contrast volume administered. One should also keep in mind that no true single value for the aortic diameter exists, since the aorta is constantly expanding and relaxing. As we applied no cardiac gating, we could not determine diastolic dimensions. It makes sense to state that the diameters obtained in vivo will probably be more representative of the systolic state whereas in vitro (casting) diameters will be a better estimate for the diastolic state. The true time-averaged diameter is most probably a value in the middle between those two. Segmentation errors also have to be taken into account, as the aortic arch is influenced by cardiac motion and is thus blurred, leading to small errors in the segmentation and the resulting $3 \mathrm{D}$ model.

When comparing the bifurcation angles (table 3) the difference between in vivo and in vitro angles is much smaller compared to the difference in diameters: only $5-20 \%$. This small difference indicates that the representation of morphological characteristics of the arterial geometry does not differ much between both imaging techniques. This can also be observed from the good colour-coded agreement between in vivo and in vitro models in figure 6, and from the correlation plot in figure 3 (left panel) and the Bland- 
Altman plot in figure 4 (left panel). The angle between the ascending and descending aorta (AA-DA in table 4) is in good agreement with previously reported measurements [21].

When comparing CFD results both spatially averaged $\mathrm{WSS}_{\mathrm{av}}$ and 95 percentile $\mathrm{WSS}_{95 \%}$ are lower for in vivo models. According to Poiseuille's law theoretical WSS values (in an infinitely long straight tube) are proportional to blood velocity and inversely proportional to diameter. Since the in vivo models are larger than the cast models but the same velocity is imposed at the inlet, it makes sense that in vivo models result in lower shear stresses. This is an important aspect that should be kept in mind when performing CFD simulations of the blood flow in murine vasculature: even when exactly the same measured velocity profile is imposed at the inlet of the model, results can be different depending on the imaging technique used to build the $3 \mathrm{D}$ model. With the casting technique, diameters will be underestimated and WSS will be overestimated. Using in vivo micro-CT, diameters will be overestimated and WSS values will be underestimated.

Keeping these remarks in mind, our results show that contrast-enhanced micro$\mathrm{CT}$ visualisation can be used to build 3D geometrical models of the aortic arch and the aortic arch bifurcations. Because of the high mortality due to the use of Fenestra VC$131^{\circledR}$, we are now using a different contrast-agent, eXIA $160 \mathrm{XL}^{\circledR}$ (Binitio Biomedical, Inc., Ottawa, Canada). eXIA $160^{\circledR}$ is a blood pool contrast agent that contains $160 \mathrm{mg}$ iodine / ml, a 3.2 times higher dose than Fenestra VC-131 ${ }^{\circledR}$. Preliminary results show that the mortality is much lower than when using Fenestra VC- $131^{\circledR}$ while preserving image quality. The decreased death rate may be due to the lower volume needed $(0.0075$ $\mathrm{ml} /$ gram) being half the dose needed when using Fenestra ${ }^{\circledR}$. The results of both contrast 
agents (using their minimal dose) are similar: both can be used to reconstruct reliable 3D models of the cardiovascular system.

\section{Conclusion}

In conclusion, with Fenestra VC-131 ${ }^{\circledR}$ it is possible to build reliable 3D geometrical models of the cardiovascular system in mice and to base CFD simulations on these models. These in vivo models have significantly larger dimensions than in vitro models based on the same geometry, also resulting in lower WSS values. However, the total morphology and bifurcation angles show only small differences, and the WSS distribution over the model surface is also very similar. In vivo micro-CT imaging thus provides a valuable alternative for vascular corrosion casting.

\section{Acknowledgments}

Research funded in part by a $\mathrm{PhD}$ grant to Bram Trachet of the Institute for the Promotion of Innovation through Science and Technology in Flanders (IWTVlaanderen). We would like to thank Philippe Joye and Steven Deleye for their help with the animal experiments. 


\section{References}

1. Daugherty, A.; Manning, M.W.; Cassis, L.A. Angiotensin II promotes atherosclerotic lesions and aneurysms in apolipoprotein E-deficient mice. J Clin Invest. 105: 1605-1612, 2000.

2. Reardon, C.A.; Getz, G.S. Mouse models of atherosclerosis. Curr Opin Lipidol. 12: $167-173,2001$.

3. Choudhury, R.P.; Aguinaldo, J.G.; Rong, J.X. et al. Atherosclerotic lesions in genetically modified mice quantified in vivo by non-invasive highresolution magnetic resonance microscopy. Atherosclerosis. 162: 315-321, 2002.

4. Dietz, H.C.; Loeys, B.; Carta, L.; Ramirez, F. Recent progress towards a molecular understanding of Marfan syndrome. Am. J. Med. Genet. C. Semin. Med. Genet. 139: 4-9, 2005.

5. Habashi, J.P.; Judge, D.P.; Holm, T.M. et al. Losartan, an AT1 antagonist, prevents aortic aneurysm in a mouse model of Marfan syndrome. Science. 312: 117-121, 2006.

6. Loeys, B.L.; Schwarze, U.; Holm, T. et al. Aneurysm syndromes caused by mutations in the TGF-beta receptor. N Engl J Med. 355: 788-798, 2006.

7. Nerem, R.M. Vascular fluid mechanics, the arterial wall, and atherosclerosis. J Biomech Eng. 114: 274-282, 1992.

8. Sawchuk, A.P.; Unthank, J.L.; Davis, T.E.; Dalsing, M.C. A prospective, in vivo study of the relationship between blood flow hemodynamics and atherosclerosis in a hyperlipidemic swine model. J Vasc Surg. 19: 58-64, 1994. 
9. Boussel, L.; Rayz, V.; McCulloch, C.; Martin, A.; Acevedo-Bolton, G.; Lawton, M. Aneurysm growth occurs at region of low wall shear stress: Patient-specific correlation of hemodynamics and growth in a longitudinal study. Stroke. 39: 2997-3002, 2008.

10. Trachet, B.; Swillens, A.; Van Loo, D. et al. The influence of aortic dimensions on calculated wall shear stress in the mouse aortic arch. Comput Methods Biomech Biomed Engin. 17, 2009.

11. Suo, J.; Ferrera, D.E.; Sorescu, D.; Guldberg, R.E.; Taylor, W.R.; Giddens, D.P. Hemodynamic shear stresses in mouse aortas: implications for atherogenesis. Arterioscler Thromb Vasc Biol. 27: 346-351, 2007.

12. Feintuch, A.; Ruengsakulrach, P.; Lin, A. et al. Hemodynamics in the mouse aortic arch as assessed by MRI, ultrasound and numerical modeling. Am J Physiol Heart Circ Physiol. 292: H884-892, 2007.

13. Mukundan, S.; Ghaghada, K.B.; Badea, C.T. et al. A liposomal nanoscale contrast agent for preclinical CT in mice. AJR Am J Roentgenol, 186: 300-307, 2006.

14. Willekens, I.; Lahoutte, T.; Buls, N. et al. Time-Course of Contrast Enhancement in Spleen and Liver with eXIA 160, Fenestra LC, and VC. Mol Imaging Biol. 11: 128-135, 2009.

15. Parker, D.L. Optimal short scan convolution reconstruction for fan beam CT. Med. Phys. 9: 254-257, 1982.

16. Ford, N.L.; Nikolov, H.N.; Norley, C.J.D. et al. Prospective respiratory-gated microCT of free breathing rodents. Med Phys. 32: 2888-2898, 2005. 
17. Ford, N.L.; Wheatley, A.R.; Holdsworth, D.W.; Drangova, M. Optimization of a retrospective technique for respiratory-gated high speed micro-CT of free-breathing rodents. Phys Med Biol. 52: 5749-5769, 2007.

18. Hodde, K.C.; Miodoński, A.; Bakker, C.; Veltman, W.A.M. Scanning electron microscopy of microcorrosion casts with special attention on the arterio-venous differences and application to the rat's cochlea. Scan Electron Microsc. 2: 477-484, 1977. 19. Sambach, S.J.; Bag, B.; Groden, C.; Schilling, L.; Brockmann, M.A. Vascular imaging in small rodents using micro-CT. Methods. DOI:10.1016/j.ymeth.2009.09.003 in press, 2006.

20. Kratky, R.G.; Roach, M.R. Shrinkage of Batson's and its relevance to vascular casting. Atherosclerosis. 51: 339-341, 1984.

21. Casteleyn, C.; Trachet, B.; Van Loo, D. et al. Validation of the murine aortic arch as a model to study human vascular diseases. J Anat. 2009.

22. Moore, J.A.; Rutt, B.K.; Karlik, S.J.; Yin, K.; Ethier, C.R. Computational blood flow modeling based on in vivo measurements. Ann Biomed Eng. 27: 627-640, 1999.

23. Hodde, K.C. Cephalic vascular patterns in the rat: A scanning electron microscopic (SEM) study of casts. Amsterdam: Rodopi; 1981:14-15, 32-33, 66. 
Table 1 Mouse specifics for 9 mice injected with Fenestra VC-131 $\left(\Delta \mathrm{T}_{\mathrm{s}}=\right.$ Time between scan and contrast injection, $\Delta \mathrm{T}_{\mathrm{c}}=$ Time between casting and scanning)

\begin{tabular}{|l|l|l|l|l|l|l|}
\hline Mouse & Age (weeks) & Weight (gr) & Contrast Dose $(\mathrm{ml} / \mathrm{gr})$ & Anesthetics $(\mathrm{ml})$ & $\Delta \mathrm{T}_{\mathrm{s}}(\mathrm{min})$ & $\Delta \mathrm{T}_{\mathrm{c}}$ \\
\hline 1 & 27.6 & 23.0 & 0.015 & 0.10 & 25 & $1 \mathrm{~h}$ \\
2 & 14 & 21.7 & 0.015 & 0.10 & 25 & $2 \mathrm{~h}$ \\
3 & 14.9 & 27.5 & 0.015 & 0.10 & 25 & $3 \mathrm{~h}$ \\
4 & 14 & 25.5 & 0.015 & 0.10 & 25 & $5 \mathrm{~h}$ \\
5 & 27.6 & 30.7 & 0.015 & 0.10 & 25 & $4 \mathrm{~h}$ \\
6 & 27.6 & 35.0 & 0.015 & 0.10 & 25 & $5 \mathrm{~h}$ \\
7 & 5 & 16.8 & 0.015 & 0.05 & 25 & $6 \mathrm{~d}$ \\
8 & 5 & 15.8 & 0.015 & 0.12 & 25 & $5 \mathrm{~d}$ \\
9 & 5 & 14.6 & 0.015 & 0.12 & 25 & $6 \mathrm{~h}$ \\
\hline
\end{tabular}

Table 2 Diameters $(\mathrm{AA}=$ Ascending Aorta; $\mathrm{BT}=$ Brachiocephalic Trunk; AoA = Aortic Arch; $\mathrm{CCA}=$ Common Carotid Artery; SA = Subclavian Artery; DA = Descending Aorta)

\begin{tabular}{|l|l|l|r|}
\hline & cast $(\mathrm{mm})$ & in-vivo $(\mathrm{mm})$ & \multicolumn{1}{c|}{ diff (\%) } \\
\hline Asc & $1.08 \pm 0.09$ & $1.43 \pm 0.07$ & 32.41 \\
BT & $0.55 \pm 0.06$ & $0.78 \pm 0.05$ & 41.82 \\
AoA & $0.93 \pm 0.05$ & $1.24 \pm 0.07$ & 33.33 \\
CCA & $0.4 \pm 0.07$ & $0.51 \pm 0.01$ & 27.50 \\
SA & $0.45 \pm 0.05$ & $0.62 \pm 0.04$ & 37.78 \\
DA & $0.87 \pm 0.09$ & $1.13 \pm 0.09$ & 29.89 \\
\hline
\end{tabular}

Table 3 Bifurcation angles $(\mathrm{BT}=$ Brachiocephalic Trunk; $\mathrm{CCA}=$ Common Carotid Artery; SA = Subclavian Artery; AA-DA = Ascending Aorta - Descending Aorta)

\begin{tabular}{|l|l|l|r|}
\hline & cast $\left({ }^{\circ}\right)$ & in-vivo $\left({ }^{\circ}\right)$ & \multicolumn{1}{c|}{ diff $(\%)$} \\
\hline BT & $136.24 \pm 6.70$ & $127.37 \pm 5.58$ & -6.51 \\
CCA & $119.56 \pm 1.64$ & $106.76 \pm 2.47$ & -10.70 \\
SA & $103.07 \pm 5.36$ & $87.29 \pm 2.80$ & -18.07 \\
AA-DA & $27.98 \pm 4.88$ & $34.79 \pm 2.63$ & 24.35 \\
\hline
\end{tabular}




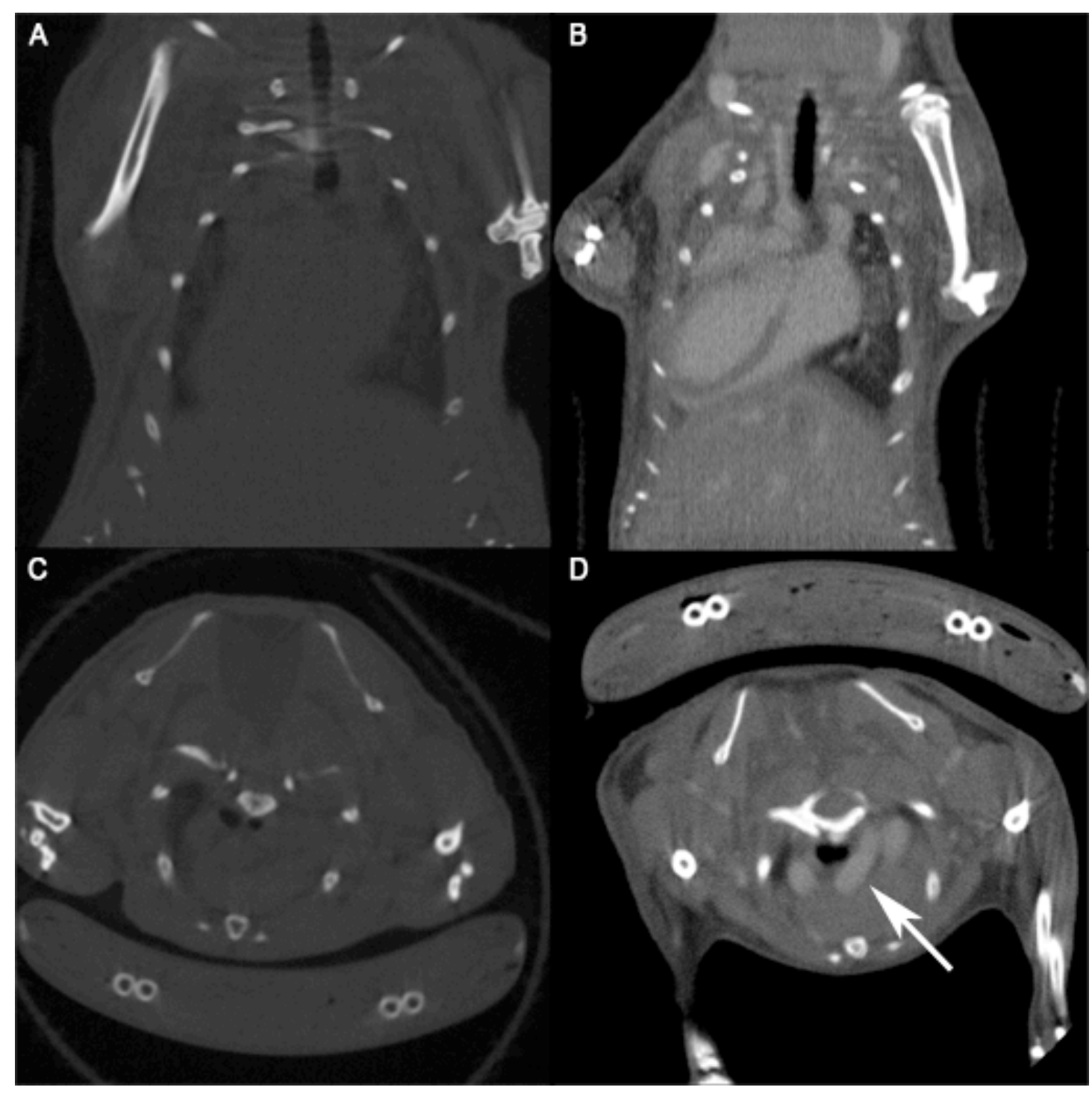

Fig. 1 Four micro-CT slices showing the difference between baseline (a and c) and contrast-enhanced imaging ( $\mathrm{b}$ and $\mathrm{d}$ ) after administration of $0.015 \mathrm{ml} / \mathrm{gram}$ Fenestra VC131. This leads to an increase of $118 \pm 2$ gray values in the aortic arch (arrow). 


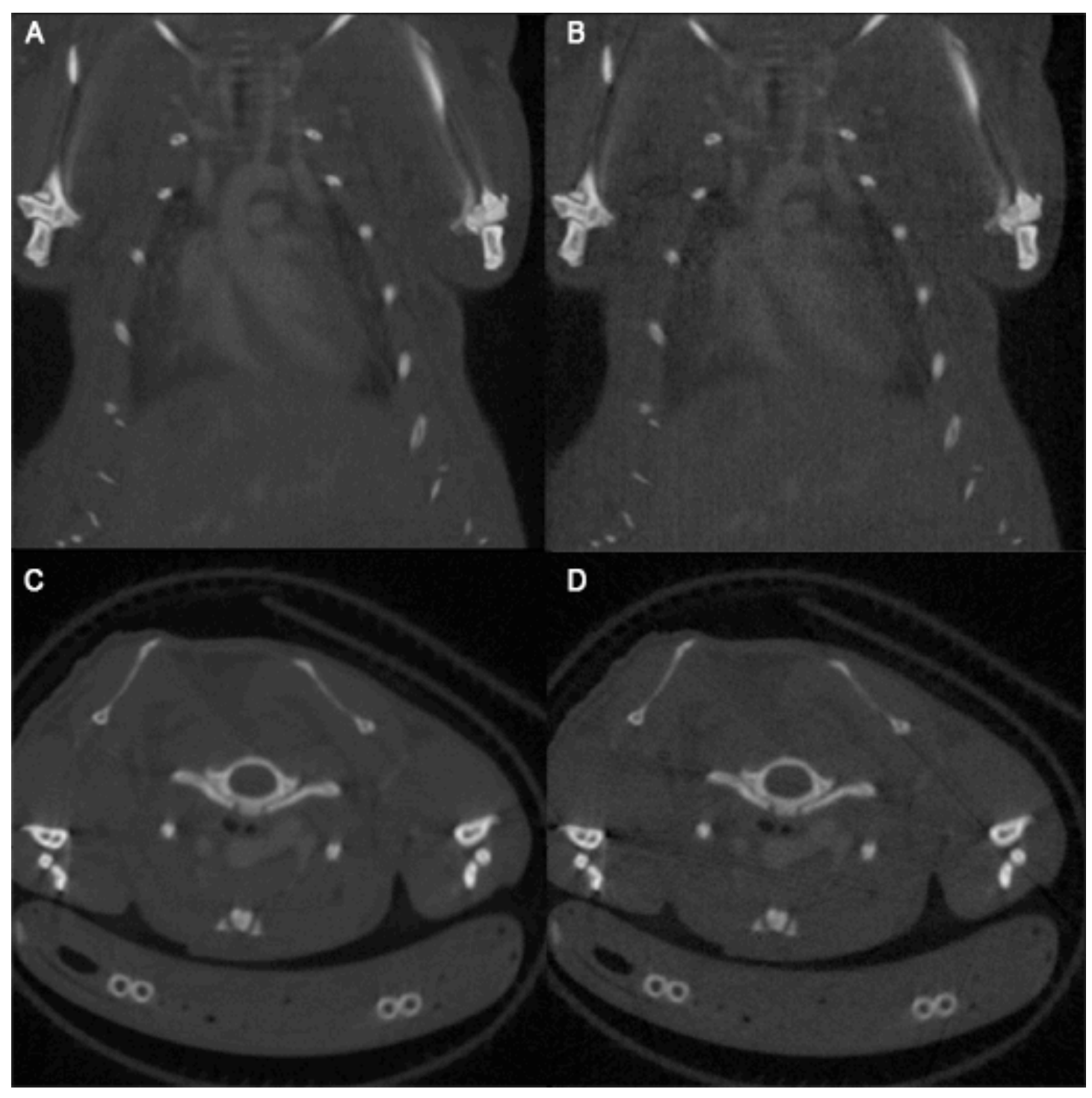

Fig. 2 Comparison between ungated slices (a and c) and retrospective respiratory-gated slices ( $b$ and $d$ ) for mouse 5 (table 1). All slices are shown in the same window level and size; The gated images show better delineation of the diaphragm and the ribs, but no significant image quality increase in the cardiovascular system 


\section{Regression Plot}

- Brachiocephalic Trunk • Common Carotid Artery - Subclavian Artery $\times$ Ascending - Descending Aorta

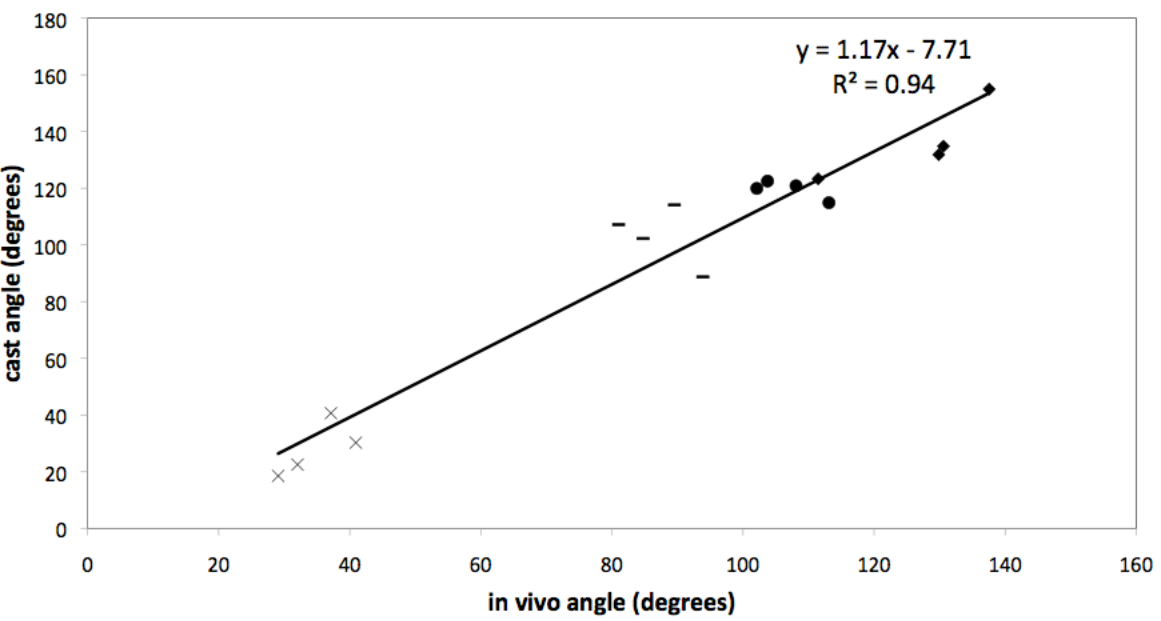

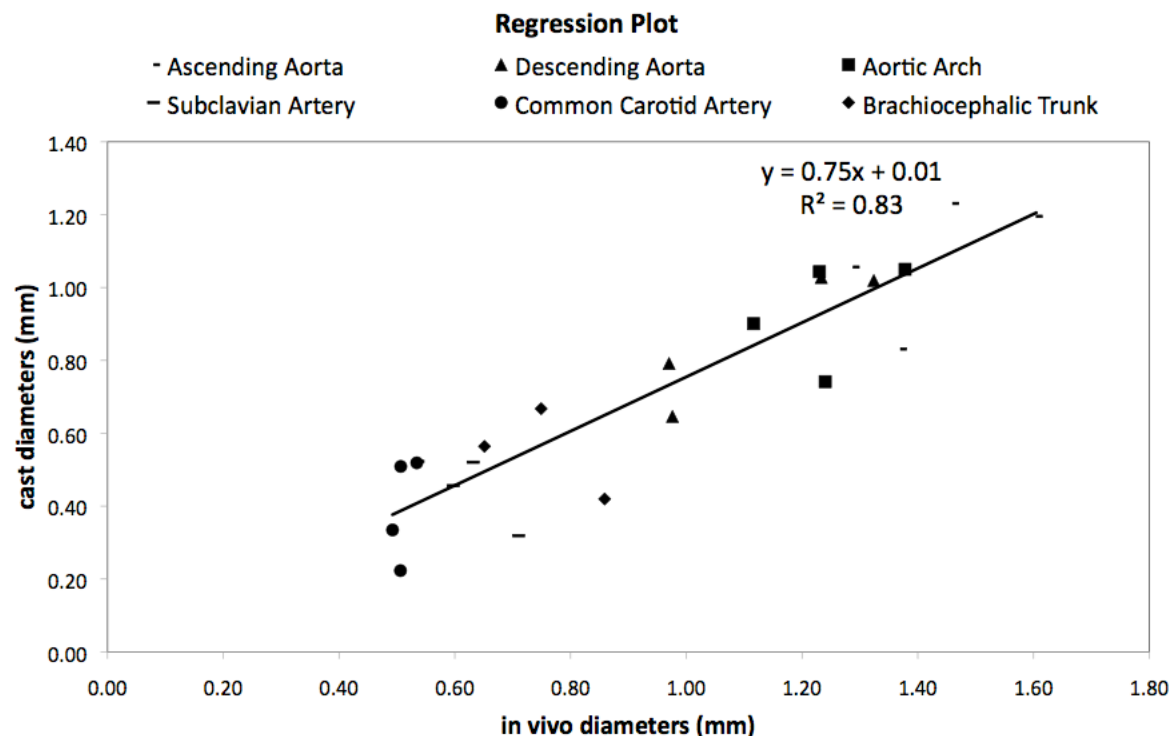

Fig. 3 Regression plots for the aortic arch angles (left) and the aortic arch diameters (right). Diameter Standard Error of the Estimate (SEE) equals $0.125 \mathrm{~mm}$, angle SEE equals 11.130 degrees. 


\section{Bland-Altman Plot}

- Brachiocephalic Trunk • Common Carotid Artery - Subclavian Artery $\times$ Ascending-Descending Aorta

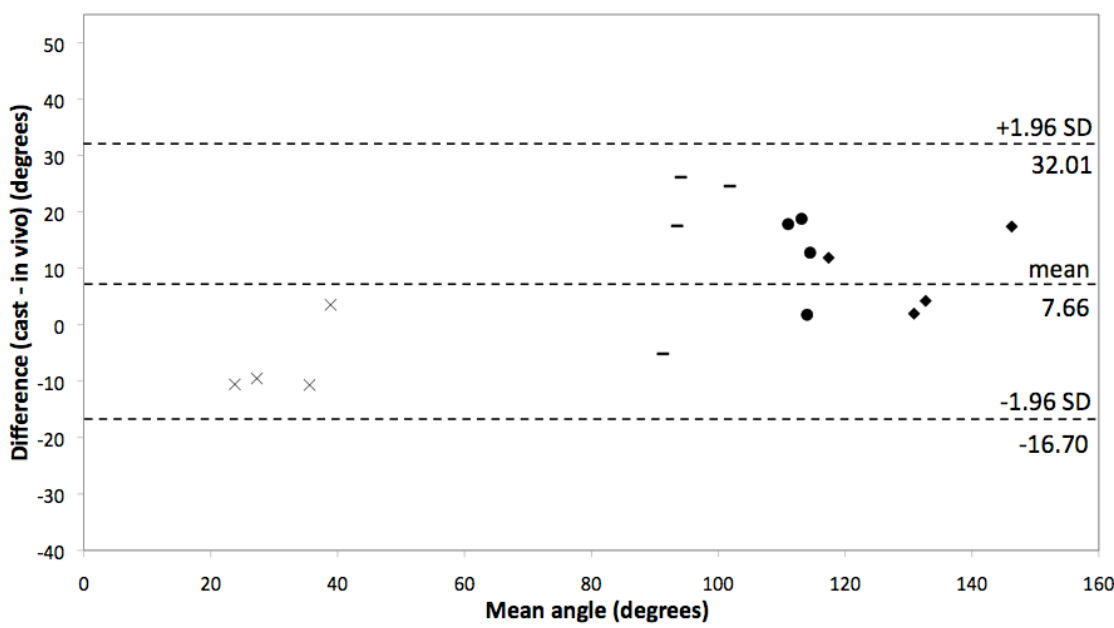

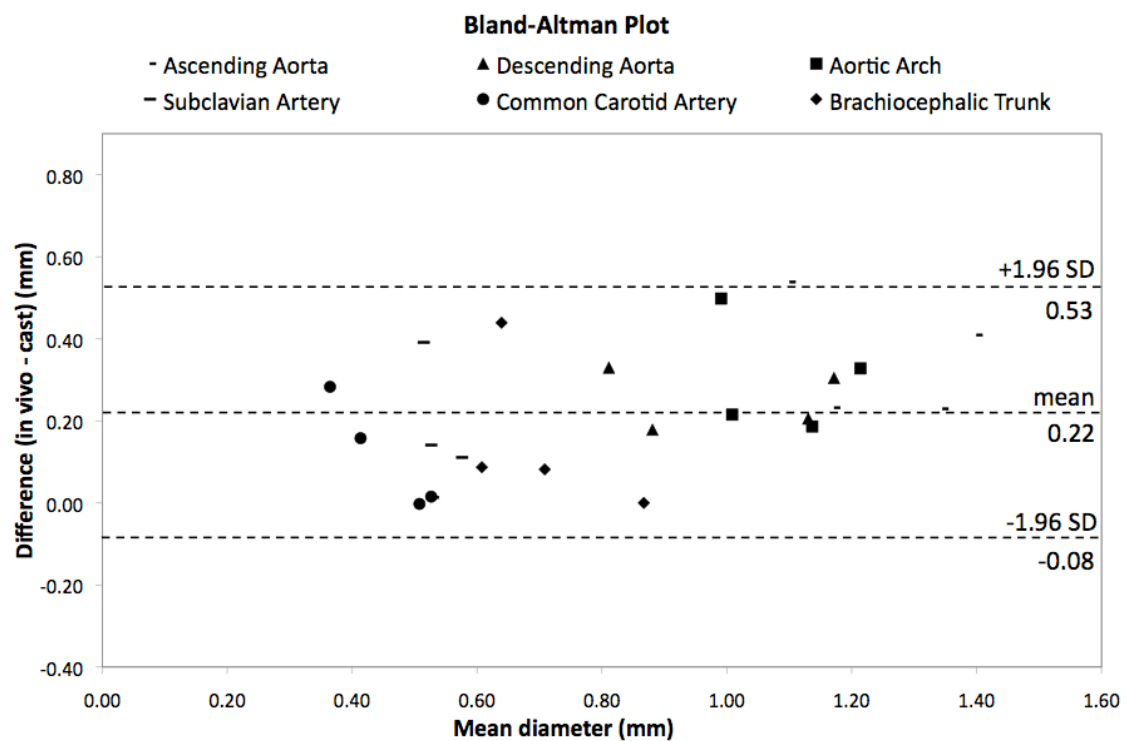

Fig. 4 Bland-Altman plots for the aortic arch angles (left) and the aortic arch diameters (right). Both plots contain the limits of agreement (average \pm 1.96 standard deviation of the difference). 


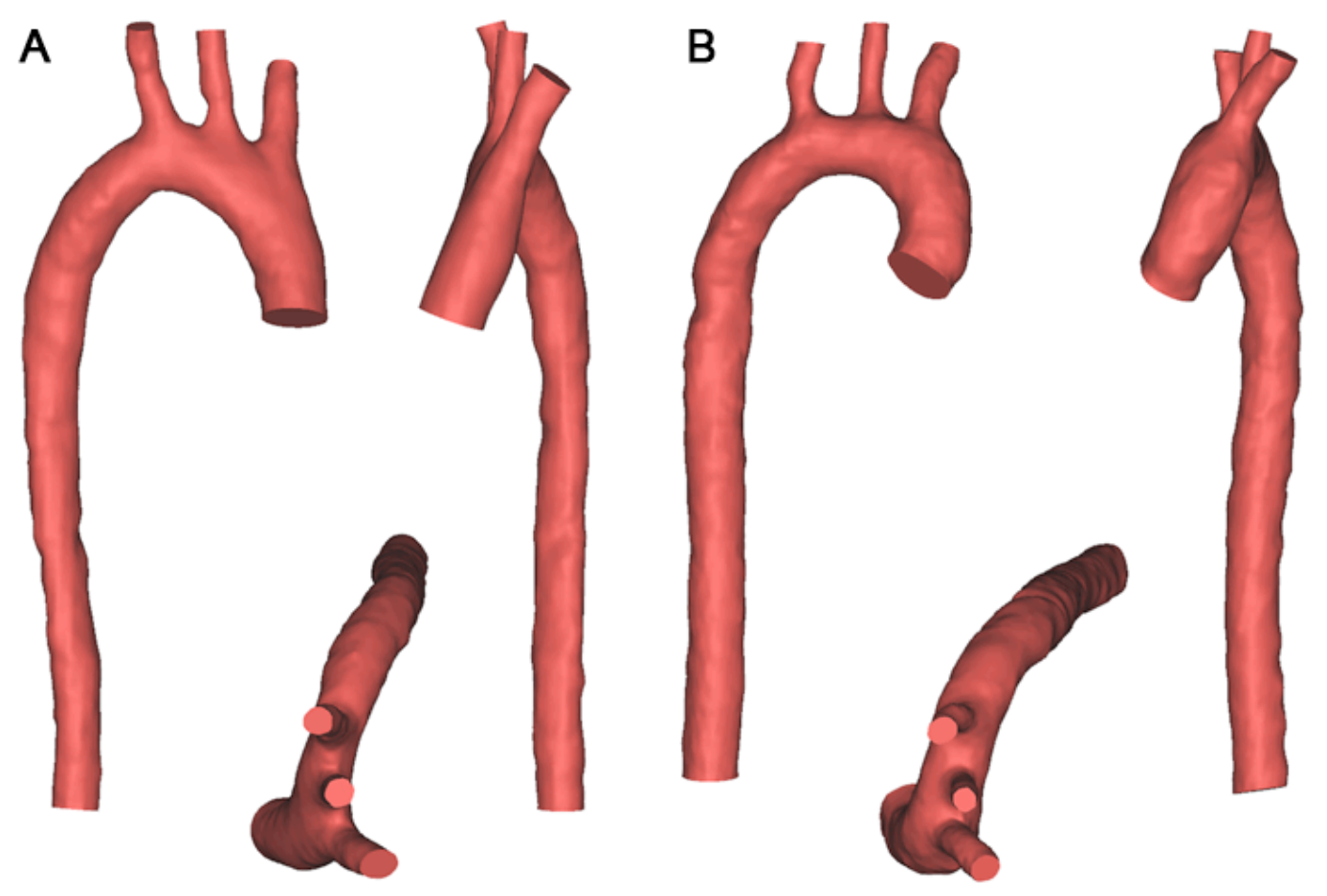

Fig. 5 General geometry of a 3D model generated from the in vivo dataset for mouse 6: cast model (a) and in vivo model (b). 

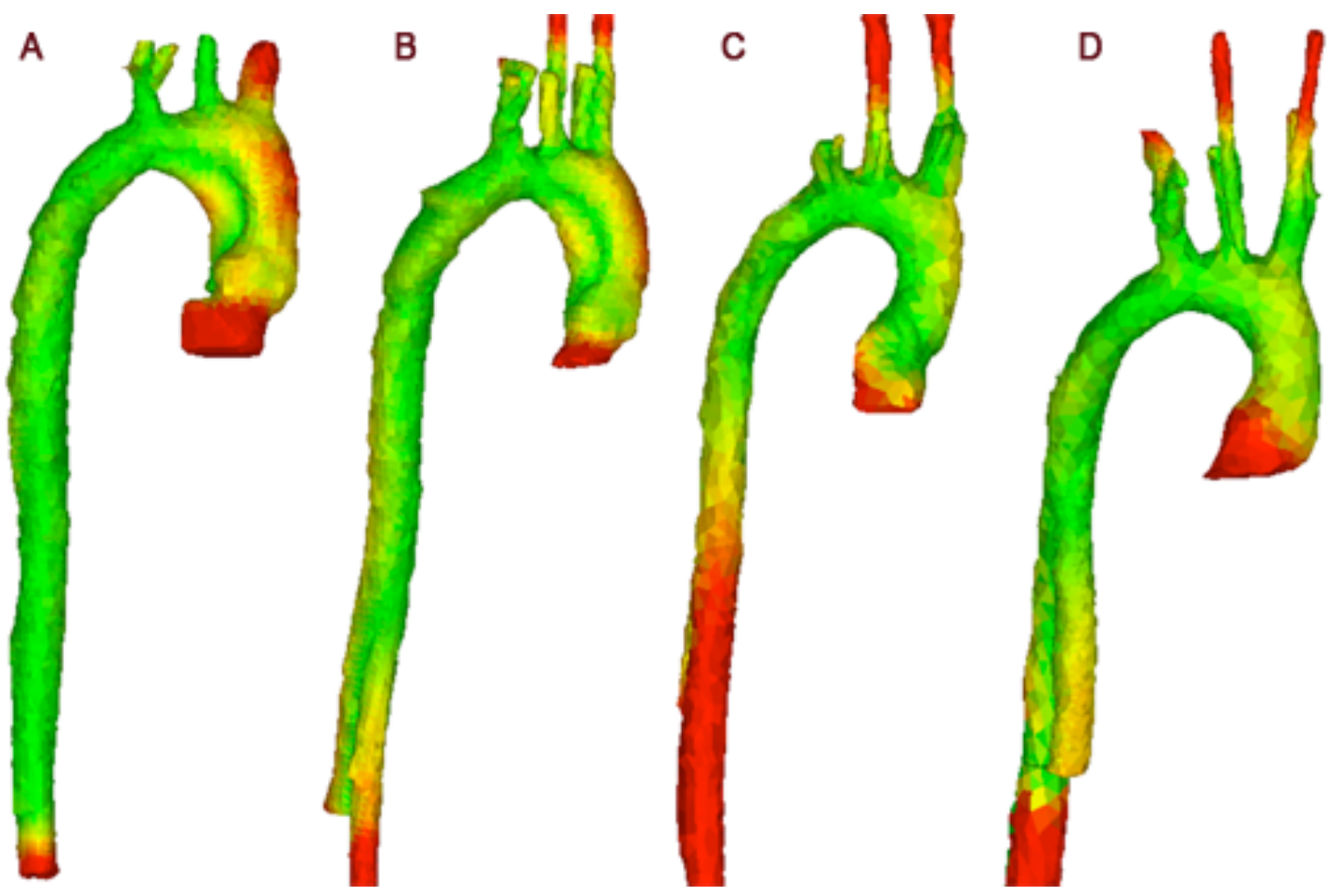

Fig. 6 Comparison between co-registered models from Fenestra VC-131 and the casts for mice 5 (a), 6 (b), 7 (c) and 8 (d). A green colour code was used to mark voxels with little distance; red was used when a large distance was measured. All colours were normalised. The descending aorta region shows the highest difference, due to the difference in AADA angles. 

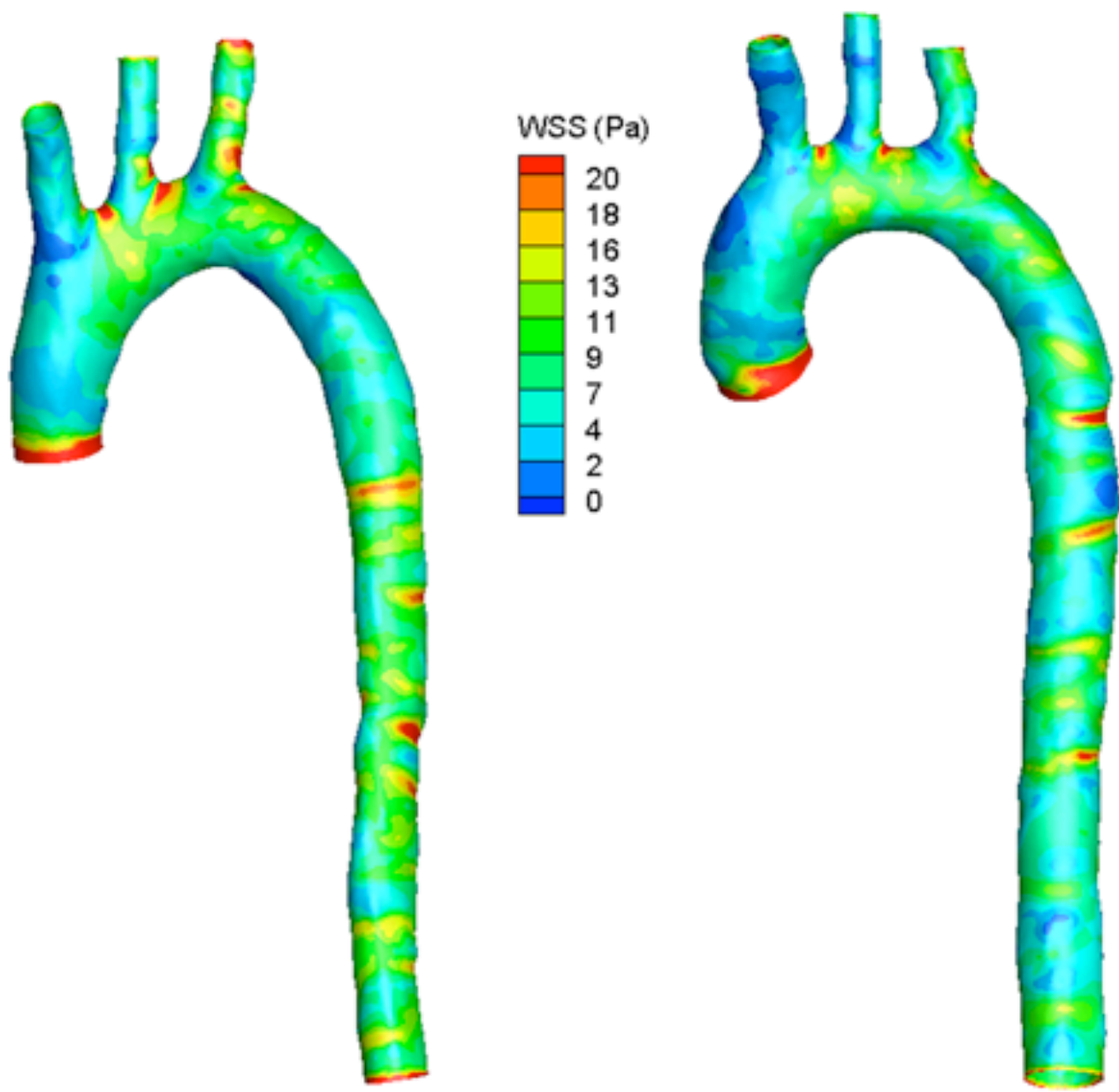

Fig. 7 Comparison of the time-averaged WSS distribution for mouse 6 for both a casting (left) and an in vivo model (right). 